\title{
A COMPARISON BETWEEN NUMERICAL DIFFERENTIATION AND KALMAN FILTERING FOR A LEO SATELLITE VELOCITY DETERMINATION
}

\author{
M.A. Sharifi, M.R. Seif*, M.A. Hadi \\ Department of Surveying and Geomatics Engineering, University College of Engineering, \\ University of Tehran, Enghelab Ave., P.O. Box 11365-4563, Tehran, Iran. \\ *Corresponding author. Email: m.r.seif@ut.ac.ir. Tel.: +98 21880088 41; fax: +98 2188 \\ 008837.
}

\begin{abstract}
The kinematic orbit is a time series of position vectors generally obtained from GPS observations. Velocity vector is required for satellite gravimetry application. It cannot directly be observed and should be numerically determined from position vectors. Numerical differentiation is usually employed for a satellite's velocity, and acceleration determination. However, noise amplification is the single obstacle to the numerical differentiation. As an alternative, velocity vector is considered as a part of the state vector and is determined using the Kalman filter method. In this study, velocity vector is computed using the numerical differentiation (e.g., 9-point Newton interpolation scheme) and Kalman filtering for the GRACE twin satellites. The numerical results show that Kalman filtering yields more accurate results than numerical differentiation when they are compared with the intersatellite range-rate measurements.
\end{abstract}

Keywords: Kalman Filtering, Numerical Differentiation, Satellite Velocity, KBR system.

\section{INTRODUCTION}

The kinematic orbit derived from GPS observations is a dense and accurate orbit. It provides necessary information for many applications in geometrical geodesy. However, for dynamic applications of satellites in geodesy, i.e. satellite gravimetry, 3D velocity and acceleration of satellites are required. Numerical differentiation is a usual method for satellite velocity and acceleration determination. Numerical differentiation is a technique to produce an estimate of the derivative of a mathematical function or function subroutine using values from the function and perhaps other knowledge about the function [1]. It is based on the approximation of the original approximating polynomial which yields the functions value at the observation points. The numerical differentiation approach used in this paper is 9-point 
Newton method. This method is a common approach in satellite geodesy for calculating velocity and acceleration of satellite [2, 3]. Numerical differentiation is based on the geometrical information without using any physical information. Amplification of the measurement noise is the main obstacle of this method [4].

Kalman filtering is a viable alternative method for deriving velocity vector from kinematic orbit. Kalman filtering is desirable in many engineering applications. The velocity vector as an unknown vector is estimated by Kalman filtering in reduced-dynamic orbit determination. Detecting gross and systematic errors and smoothing random error are the most important advantages of this method $[5,6,7]$.

Incorporating dynamic model as a function with physical properties in addition to geometrical information, kinematic orbit, into Kalman filtering process is the superior reason of this approach in comparison with numerical differentiation. By using dynamic model, the effects of geometrical errors, noise, systematic and gross errors, will be reduced. Unlike Kalman filtering, gross and systematic errors will be directly entered into results of numerical differentiation methods and noise will be amplified.

The K-Band Ranging (KBR) observations of twin satellites GRACE are used for comparison between velocity vectors derived from numerical differentiation and Kalman filtering. The ranging system precisely measures the distance changes between the twin satellites in time. This precise measurement is a reliable reference for a proper comparison between velocity vectors of both methods. The results show the reduced dynamic velocity vector is more accurate than the velocity vector obtained by numerical differentiation.

\section{METHODOLOGY}

As an alternative strategy, velocity vector was estimated using extended Kalman filtering instead of numerical differentiation. Therefore, these methods are concisely described in the following sub-sections.

\subsection{Numerical differentiation}

Kinematic orbit is a time series of satellite positions. However, velocity vector is required for more applications e.g. in gravity field recovery using the energy integral [8]. The numerical differentiation is a mathematical process for computing the numerical value of derivative of a function [9]. Newton interpolation is suitable and highly efficient for many applications in mathematics e.g. in numerical integration, numerical differentiation and polynomial approximation. This method could be used for evaluating numerical value of derivative of a given function by deriving of approximated polynomial. The n-point Newton interpolation formula, for equidistant sampling points is [2]:

$$
\begin{aligned}
& f(t)=f\left(t_{1}+\sigma h\right)=f\left(t_{1}\right)+\left(\begin{array}{c}
\sigma \\
1
\end{array}\right) \Delta_{3 / 2}^{1}+\left(\begin{array}{l}
\sigma \\
2
\end{array}\right) \Delta_{2}^{2} \\
& +\ldots+\left(\begin{array}{c}
\sigma \\
n-1
\end{array}\right) \Delta_{1+(n-1) / 2}^{n-1} \quad, \sigma \in[0, n-1]
\end{aligned}
$$


with Newton interpolation forward differences

$$
\Delta_{1+(n-1) / 2}^{n-1}=\sum_{i=1}^{n}(-1)^{n+i}\left(\begin{array}{c}
n-1 \\
i-1
\end{array}\right) f\left(t_{i}\right)
$$

where $f\left(t_{1}\right)$ is initial value of function, $h=t_{i+1}-t_{i}$ is the sampling interval, and $\sigma$ is defined as the normalized distance $\sigma=\left(x-x_{1}\right) / h$. In the n-point interpolation scheme, the central point $t_{(n+1)}$ has the most optimal performance. By deriving of this interpolated polynomial, the first-, second- and higher order derivatives could be obtained.

\subsection{Extended Kalman Filtering}

Kalman filtering is a recursive filtering that each state is computed from the previous state and the new observation, so only the previous estimate requires storage [10]. The relations of Kalman filtering are based on minimizing the variance of the estimation error.

Kalman filtering algorithm is useable for linear dynamic system with linear observation equations. By linearizing nonlinear system, a new error source is entered to computation process named linearization error. Although the linearized Kalman filtering is adequate for many applications, it may not be adequate in orbit determination process, due to high nonlinearity of equations of motion [11]. Therefore to minimize the effects of errors due to the neglect of higher order terms in the linearization procedure, extended Kalman filtering was selected instead of standard Kalman filtering in presented paper. The extended form of the sequential estimation algorithm is [12]:

$$
\begin{gathered}
X_{i+1}^{-}=f\left(X_{i}^{+}, t_{i}\right) \\
P_{i+1}^{-}(t)=\Phi\left(t_{i+1}, t_{i}\right) P_{i}^{+} \Phi^{T}\left(t_{i+1}, t_{i}\right)+Q_{i}
\end{gathered}
$$

The time-update phase updates the state $X_{i}^{+}$and the covariance matrix $P_{i}^{+}$along the time using the dynamical model $f\left(X_{i}^{+}, t_{i}\right)$. Where $Q_{i}$ is the covariance of the process noise and $\Phi\left(t_{i+1}, t_{i}\right)$ is the state transition matrix, which relates the state deviation between $t_{i+1}$ and $t_{i}$. The state transition matrix is directly determined using numerical integration of the matrix differential equation by considering gravitational and non-gravitational forces acting on satellite.

In the measurement update phase, the estimated state vector $X_{i+1}^{+}$and the estimated error covariance $P_{i+1}^{+}$are given by Eq. (5) and (6), respectively:

$$
X_{i+1}^{+}=X_{i+1}^{-}+K_{i+1} \delta y_{i+1}
$$




$$
P_{i+1}^{+}=\left(I-K_{i+1} H_{i+1}\right) P_{i+1}^{-}
$$

where $K_{i+1}$ is Gain matrix obtained by

$$
K_{i+1}=P_{i+1}{ }^{-} H_{i+1}{ }^{T}\left(H_{i+1} P_{i+1}{ }^{-} H_{i+1}{ }^{T}+R_{i+1}\right)^{-1}
$$

where $\delta y_{i+1}, R_{i+1}$ and $H_{i+1}$ respectively are the observation residual, the covariance matrix of observation noise, and the design matrix of the observation.

\subsubsection{Observation Model}

Different type of observations can be used for estimation of satellite positions as the output of kinematic orbit determination process. In this research for simplicity of implementation, code measurements are used for estimation of LEO satellites kinematic orbits. The observed orbit is obtained from ionosphere-free linear combination of the GPS satellite P-code.

\subsubsection{Dynamic Model}

The dynamic model described the satellite's motion with time was constructed using forces acting on the satellite. The gravitational forces such as geopotential, gravity of the Sun and Moon and indirect effect of third body, solid and ocean tide, and non-gravitational forces such as atmospheric drag and solar radiation pressure have been modeled for transition matrix determination in orbit determination process.

In this paper, EGM96 was applied as a gravity field of Earth up to the 70th order and degree [13]. The ephemerides of the Moon and Sun are respectively calculated using the theory ELP-2000/82 represented by Chapront-Touze and Chapront [14] and analytical formulas [15]. A dynamic global of the Earth's atmosphere, NRLMSISE-00, was used for the air-drag force computations [16]. The solar radiation pressure is obtained from the calculation of the sunlight percentage [17]. IERS formulations are used for solid Earth tide modeling [18].

\section{NUMERICAL ANALYSIS}

The K-Band Ranging (KBR) system measures the variation of distance between the GRACE twin satellites, by the precision of $1 \mu \mathrm{m} / \mathrm{sec}$ [19]. Due to high precision of range rate obtained from KBR system, this observation could be used as a reference for qualify control of kinematic and reduced-dynamic orbits. The satellites range-rate is a function of position and velocity vectors of the GRACE satellite pairs. Comparing the computed and observed range-rate measurements, the quality of kinematic or reduced-dynamic orbits is determined. The relation between inter satellite range-rate and position and velocity vectors of twin satellite is [20]:

$$
\dot{\boldsymbol{\zeta}}=\frac{\left(\underline{r}_{A}-\underline{r}_{B}\right) \cdot\left(\underline{\dot{r}}_{A}-\underline{\dot{r}}_{B}\right)}{\left|\underline{r}_{A}-\underline{r}_{B}\right|}
$$


$\underline{r}_{A}$ and $\underline{r}_{B}$ are the position vectors of GRACE A and B where $\underline{r}_{A}$ and $\underline{r}_{B}$ show their respective velocity vectors.

The numerical differentiation is used for calculation of the velocity vectors required for computation of the range-rate computed values. For quality control of the reduced-dynamic and kinematic orbits, the computed range-rate obtained from these orbits are compared by the observed value. The algorithm of this comparison is shown in figure 1.

In the first approach, both the position and velocity vectors needed for range-rate computation are obtained via filtering. In the second approach position vector is obtained from kinematic orbit and velocity vector is computed using numerical differentiation. In the third approach, the position vector is obtained from Kalman filtering and velocity vector is computed from numerical differentiation. The third approach is a combination of filtering and numerical differentiation. In this way, both approaches, the first and third, use the filtered positions. The only differences are due to the error of the velocity vectors. Therefore, the comparison between the computed range-rate resulted from these two approaches with the observed quantity is equivalent to comparison of the velocity vectors achieved from Kalman filter/Numerical differentiation methods.

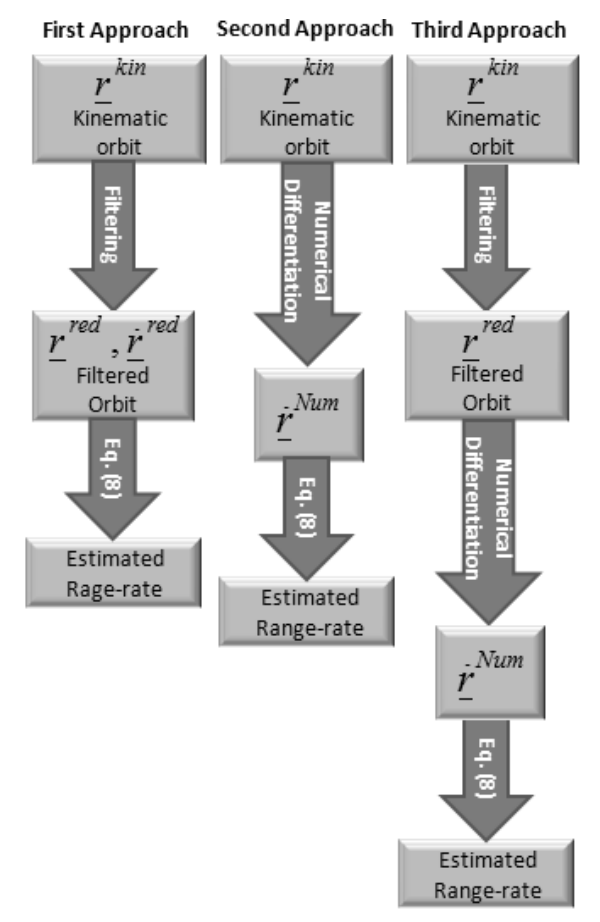

Fig. 1. Algorithm for range-rate comparison

Differences between the observed and computed range-rates from three approaches are plotted in Figure 2. 


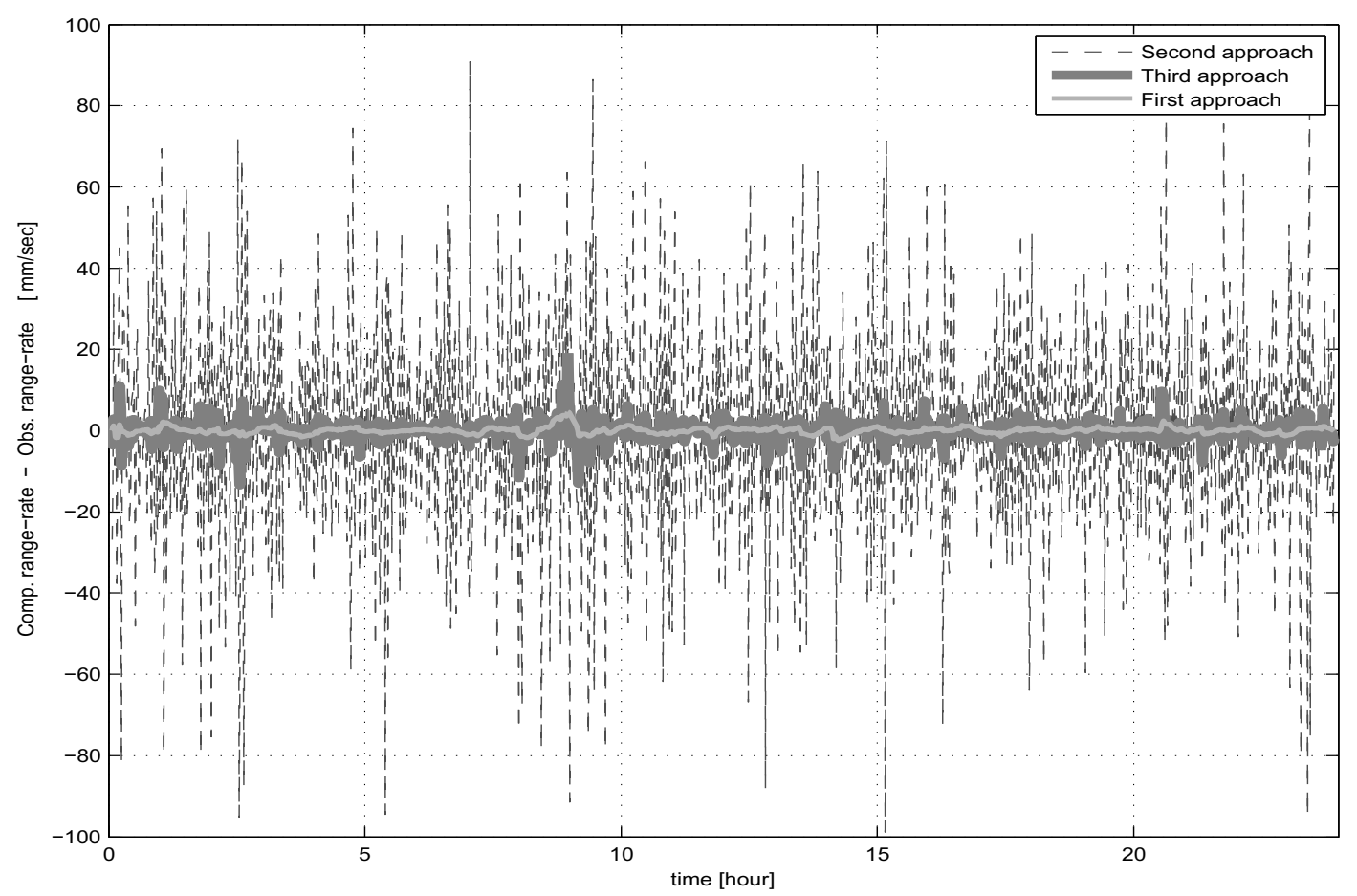

Fig. 2. The differences between the observed and computed range-rate from the first (lightest), second (light) and third (dark) approaches

The lightest and light curves show the difference between the computed range-rate from the kinematic orbits (with and without filtering) from the observed range-rate respectively. It is clear that the filtered position vectors using the Kalman procedure lead to more accurate results.

The dark curves show differences between the observed and computed range-rate from the third approach. As seen, the computed range-rate derived from first approach is better than that of the third too. However, the only difference in both approaches is the velocity vectors. As a result, it could strictly be said that the quality of the velocity vector derived from Kalman filtering is much better than the velocity vector derived from the numerical differentiation method (e.g., 9-point Newton differentiation).

Table 1 shows the basic statistics of the differences between the observed and computed range-rate from three approaches of range-rate comparison algorithm.

Table 1. The basic statistics of range-rate comparisons from three approaches

\begin{tabular}{lllll}
\hline Approach & $\begin{array}{l}\min \\
(\mathrm{mm} / \mathrm{s})\end{array}$ & $\begin{array}{l}\max \\
(\mathrm{mm} / \mathrm{s})\end{array}$ & $\begin{array}{l}\text { mean } \\
(\mathrm{mm} / \mathrm{s})\end{array}$ & $\begin{array}{l}\text { std } \\
(\mathrm{mm} / \mathrm{s})\end{array}$ \\
\hline 1st App. & -2.49 & 4.70 & 0.03 & 0.81 \\
2nd App. & -658.01 & 281.74 & -0.01 & 38.72 \\
3rd App. & -13.41 & 19.15 & 0.00 & 2.33 \\
\hline
\end{tabular}




\section{CONCLUSIONS}

Comparison between the numerically derived velocity vector and that of the Kalman filtering shows that the velocity vector can be derived with lower noise level in Kalman filtering. Higher accuracy can be obtained even if the kinematic orbit is filtered before the implementation of numerical differentiation. Velocity enhancement with the filtered orbit is due to reduction of the effects of the geometrical errors (observation noise, systematic and gross errors) on the purely kinematic orbit.

\section{REFERENCES}

[1] L. Richard, J. Burden, F. Douglas, Numerical Analysis (7th Ed), Brooks, Cole, 2000.

[2] T. Reubelt, G. Asusten, E.W. Grafarend, Harmonic analysis of the Earth's gravitational field by means of semi-continuous ephemerides of a low Earth orbiting GPS-tracked satellite. Case study: CHAMP. J Geod 77 (2003) (5-6):257-278. DOI : 10.1007/s00190003-0322-9

[3] L. Foldvary, D. Svehla, Ch. Gerlach, M. Wermuth, Th Gruber, R. Rummel, M. Rothacher, B. Frommknecht, Th. Peters, P. Steigenberger, Gravity model TUM-2sp based on the energy balance approach and kinematic CHAMP orbits, Earth Observation with CHAMP Results from Three Years in Orbit (Ed. Reigber Ch, Lühr H, Schwintzer P et al.), Springer, Berlin, 13-18, 2004.

[5] P.S. Maybeck, Stochastic Models, Estimation, and Control. Volume 1, Academic Press, Inc, 1979.

[4] M. Hanke, O. Scherzer, Inverse problems light: numerical differentiation. Amer. Math. Monthly, 108 (2001) 512-5211.

[6] A. Gelb, Applied Optimal Estimation. MIT Press, Cambridge, MA, 1974.

[7] R.G. Brown, P.Y.C Hwang, Introduction to Random Signals and Applied Kalman Filtering: With MATLAB Exercises and Solutions, 3rd ed., Wiley, New York, 1997.

[8] L. Foldavary, Analysis of Numerical Differentiation methods Applied for Determination of Kinematic Velocities for LEOs, Per. Pol. Civil Eng., 51/1 17-24, 2007.

[9] W.H. Press, B.P. Flannery, S.A. Teukolsky, W.T. Vetterling, Numerical Recipes in FORTRAN. The Art of Scientific Computing, 2nd ed, Cambridge University Press, Cambridge, 1992.

[10] R.E. Kalman, A new approach to linear filtering and prediction problems. Transactions of the ASME, Ser. D, Journal of Basic Engineering, 82 (1960) 34-45.

[11] D.A. Vallado, Fundamentals of Astrodynamics and Applications. Third Edition. Published Jointly By Microcosm and Springer, New York, 2007.

[12] B.D. Tapley, B.E. Schutz, G.H. Born, Statistical Orbit Determination. Elsevier Academic Press, New York, 2004

[13] F.G. Lemoine, et al., The development of the joint NASA GSFC and the National Imagery Mapping Agency (NIMA) geopotential model EGM96. NASA Technical Report NASA/TP-1998-206861, Goddard Space Flight Center, Greenbelt, Maryland, 1998.

[14] M. Chapront-Touze, J. Chapront, The lunar ephemeris ELP 2000, Astronomy and Astrophysics, 190 (1988) 342-352.

[15] O. Montenbruck, Practical Ephemeris Calculations, Springer Verlag, Heidelberg, 1989. 
[16] J.M. Picone, A.E. Hedin, D.P. Drob, A.C. Aikin, NRLMSISE-00 empirical model of the atmosphere: Statistical comparisons and scientific issues. J. Geophys. Res., 107 (2002). doi:10.1029/2002JA009430, 2002.

[17] O. Montenbruck, E. Gill, Satellite orbits - models, methods, and applications. Springer, Berlin, 2000.

[18] D.D. McCarthy, G. Petit, IERS Conventions, IERS Tech. Note, vol. 32. Verlag des Bundesamts fur Kartogr. und Geod., Frankfurt am Main, Germany, 2004. Available at: http://www.iers.org/iers/publications/tn/tn32

[19] Z. Kang, B. Tapley, S. Bettadpur, J. Ries, P. Nagel, R. Pastor, Precise orbit determination for the GRACE mission using only GPS data, J. Geod 80 (2006)322-331.

[20] K. Case, G. Kruizinga, S. Wu, GRACE level 1B Data Product User Handbook Version 1.2, 2004.

Received: 2013-02-24,

Reviewed: 2013-03-05, by S. Oszczak,

Accepted: 2013-03-12. 